\title{
Synthesis and Characterization of novel N-(benzo[d]thiazol-2-yl)-2-(2-(6- chloroquinolin-4-yl)hydrazinyl)acetamide derivatives containing Quinoline linkage as potent antibacterial agents
}

\author{
Manoj N. Bhoi, Mayuri A. Borad, Hitesh B. Parmar and Hitesh D. Patel \\ Department of Chemistry, School of Sciences, Gujarat University, Ahmedabad, India. \\ E-mail address: hitesh13chem@rediffmail.com
}

Keywords: Antibacterial activity, Benzothiazole, chloroacetylchloride, heterocyclic compound, Quinolines

\begin{abstract}
As part of ongoing studies in developing new antibacterial agent, a novel series of N(benzo[d]thiazol-2-yl)-2-(2-(6-chloroquinolin-4-yl)hydrazinyl)acetamide derivatives was synthesized by sequencing reaction of 2-aminobenzothiazole with chloroacetylchloride, hydrazine hydrate and 4,7-dichloroquinoline. The novel heterocycles were characterized by elemental analyses and various spectroscopic techniques. The synthesized compounds were tested in-vitro antibacterial activity against two Gram-positive and two Gram-negative bacteria with standard drugs. Microbiological results showed that the synthesized compounds possessed a broad spectrum of antibacterial activity against the tested microorganisms.
\end{abstract}

\section{INTRODUCTION}

For more than an era, heterocycles have constituted one the largest areas of research in the field of organic chemistry. They have contributed to the development of society from a biological and industrial point of view. Heterocycles have played an important role in biochemical processes since the side groups of the most typical and essential constituents of living cells, DNA and RNA, are based on aromatic heterocycles.[1] Among the approximately 20 million chemical compounds identified by the end of the second millennium, more than two-thirds are fully or partially aromatic, and approximately half are heterocyclic. Between them, sulfur and nitrogen-containing heterocyclic compounds have maintained the interest of researchers through decades of historical development of organic synthesis. The grounds of this interest were their biological activities and unique structures that led to several applications in different areas of pharmaceutical and agrochemical research or, more recently, in material sciences.[2]

Benzothiazole is one of the infrequently obtainable heterocyclic compound, which found in alkaloids. It derived from terrestrial and marine natural products. Benzothiazole are an important class of bioactive and industrially important organic compounds. From the recent literature, these derivatives are also known to possess anti-tubercular,[3] local anesthetic,[4] antidiabetic,[5] antiulcer,[6] antipsychotic,[7] anti-tumor,[8] anti-oxidant,[9] analgesic,[10] schistosomicidal,[11] antileishmanial, [12] anti-convulsant,[13] anti-parasitic,[14] diuretic,[15] plant growth regulator [16] activities, anthelmintic,[17] Acaricidal.[18]

Quinoline or 1-aza-naphthalene is one of the most important N-based heterocyclic aromatic compounds. Quinolines recently have been caught the attention of researchers owing to their broad range of activities and of course for their wide applications such as Antimalarial, Anti-inflammatory activity, Analgesic activity, Antineoplastic, Antibacterial, Antifungal, Anthelmintic, Antiviral, Antiprotozoal, Cardiovascular activity, CNS effects, Hypoglycaemic activity, Reproductive System etc.[19]

There are very scarce recent literature data on antimicrobial potentials of benzothiazole containing quinolines linkage that should combine favorable structural properties of both quinolines and benzothiazole moiety. Therefore, in the present paper, we have prepared a set of new N(benzo[d]thiazol-2-yl)-2-(2-(6-chloroquinolin-4-yl)hydrazinyl)acetamide derivatives and evaluated for their in-vitro antibacterial activities against Gram-positive and Gram-negative bacteria. 


\section{EXPERIMENTAL SECTION}

All Starting materials and other reagents were purchased from commercial suppliers and were used without any further purification unless otherwise indicated. The reactions were assayed by thin layer chromatography (TLC) and terminated as judged by the consumption of starting material. Analytical thin-layer chromatography (TLC) was performed on silica gel G $60 \mathrm{~F}_{254}$ (Merck) plates and eluted with the appropriate solvent ratios $(\mathrm{v} / \mathrm{v})$. The melting points were recorded on optimelt automated melting point system and were uncorrected. IR spectra was recorded on a Perkin-Elmer 377 spectrophotometer, ${ }^{1} \mathrm{H}$ NMR spectra was measured on Bruker AV $400 \mathrm{MHz}$ using DMSO as a solvent and TMS as an internal standard. Mass spectra was recorded on Advion Expression CMS, USA. Elemental analysis were performed on the vario MICRO cube, elementar CHN analyser serial no.: 15084053.

\section{General process of 2-Chloro-N-(benzothiazol-2-yl)acetamide derivative (2a-2g):}

This compound was prepared by adding chloroacetyl chloride $(0.01 \mathrm{~mol})$ dropwise with stirring to a mixture of 2-amino-benzothiazole 1 derivative $(0.01 \mathrm{~mol})$ and triethylamine $(0.1 \mathrm{~mL})$ in $\mathrm{CHCl}_{3}(20$ $\mathrm{mL}$ ). The reaction mixture was heated under reflux for $12 \mathrm{~h}$, then the solvent was evaporated under reduced pressure and the remaining solid was crystallized.[20]

\section{General process of $\mathrm{N}$-(benzothiazol-2-yl)-2-hydrazinylacetamide derivative (3a-3g):}

Derivative of N-(benzothiazol-2-yl)-2-hydrazinylacetamide was prepared by refluxing a mixture of compound 2a-2g $(0.01 \mathrm{~mol})$ and hydrazine hydrate $99 \%(0.1 \mathrm{~mol})$ in ethanol $(30 \mathrm{~mL})$ for $14 \mathrm{~h}$. The reaction mixture was cooled, and the precipitated solid was collected by filtration, washed with water, dried, and crystallized.

\section{General process of synthesis of (5a-5g):}

A solution of $\mathrm{N}$-(benzothiazol-2-yl)-2-hydrazinylacetamide derivative (3a-3g) $(0.01 \mathrm{mmol})$ and 4,7 dichloroquinoline $(0.01 \mathrm{mmol})$ taken in $25 \mathrm{ml}$ flat bottom flask with alcoholic $\mathrm{K}_{2} \mathrm{CO}_{3}(5-10 \mathrm{ml})$. The resulting mixture was put in the heated oil bath for 6-8 hrs at reflux temperature. The time taken by different derivative of 2 -amino benzothiazole in the reaction is shown in Table 1 . After confirmation of each reaction using TLC analysis (30\% Ethyl acetate: hexane), the reaction mixture was allowed to cool at room temperature and poured into cold water. The mixture was filtered continuously along with a wash of water. The solid crude product was simply purified by column chromatography over silica gel using a solvent system (30\% ethyl acetate: hexane) to obtain subsequent pure product (5a-5g) with $65-75 \%$ yield.

\section{N-(benzo[d]thiazol-2-yl)-2-(2-(6-chloroquinolin-4-yl)hydrazinyl)acetamide (5a):}

IR $(\mathrm{KBr})\left(\mathrm{v}_{\max }, \mathrm{cm}^{-1}\right): 3070(\mathrm{C}-\mathrm{H}$ Aromatic), $3034(\mathrm{C}-\mathrm{H}$ alkene), $1640(\mathrm{C}=\mathrm{C}$ alkene $), 1540(\mathrm{C}=\mathrm{C}$ aromatic), $1670\left(\mathrm{C}=\mathrm{O}\right.$ amide), 1390 (C-N Aromatic); ${ }^{1} \mathrm{H}$ NMR (400 MHz, DMSO) $\delta 8.69(\mathrm{~d}, 1 \mathrm{H})$, $8.62(\mathrm{~s}, 1 \mathrm{H}), 8.47-7.73(\mathrm{~m}, 3 \mathrm{H}), 7.63(\mathrm{dd}, 1 \mathrm{H}), 7.58-7.45(\mathrm{~m}, 3 \mathrm{H}), 6.73(\mathrm{~d}, 1 \mathrm{H}), 3.68(\mathrm{~s}, 1 \mathrm{H})$, $3.58(\mathrm{~s}, 1 \mathrm{H}), 3.22(\mathrm{~s}, 1 \mathrm{H}), 1.83(\mathrm{~s}, 1 \mathrm{H})$; ESI-MS: $\mathrm{m} / \mathrm{z}$ Calculated 383.85, found $[\mathrm{M}+\mathrm{H}]^{+} 384.8$; Anal. Calcd for $\mathrm{C}_{18} \mathrm{H}_{14} \mathrm{ClN}_{5} \mathrm{OS}$ : C, 56.32; H, 3.68; Cl, 9.24; N, 18.24; O, 4.17; S, 8.35; found C, $56.56 ; \mathrm{H}, 3.89 ; \mathrm{N}, 18.45 ; \mathrm{S}, 8.59$.

\section{2-(2-(6-chloroquinolin-4-yl)hydrazinyl)-N-(6-methylbenzo[d]thiazol-2-yl)acetamide (5b):} IR $(\mathrm{KBr})\left(\mathrm{v}_{\max }, \mathrm{cm}^{-1}\right): 3050(\mathrm{C}-\mathrm{H}$ Aromatic), $3042(\mathrm{C}-\mathrm{H}$ alkene $), 1670(\mathrm{C}=\mathrm{C}$ alkene $), 1570(\mathrm{C}=\mathrm{C}$ aromatic), $1676\left(\mathrm{C}=\mathrm{O}\right.$ amide), 1280 (C-N Aromatic); ${ }^{1} \mathrm{H}$ NMR (400 MHz, DMSO) $\delta 10.71(\mathrm{~s}, 1 \mathrm{H})$, $8.57(\mathrm{~d}, 1 \mathrm{H}), 8.07(\mathrm{~d}, 1 \mathrm{H}), 7.97(\mathrm{dd}, 2 \mathrm{H}), 7.58-7.49(\mathrm{~m}, 2 \mathrm{H}), 7.37(\mathrm{dd}, 1.4 \mathrm{~Hz}, 1 \mathrm{H}), 6.68(\mathrm{~d}, 1 \mathrm{H})$, $3.56(\mathrm{~s}, 1 \mathrm{H}), 3.49(\mathrm{~d}, 2 \mathrm{H}), 3.08(\mathrm{~s}, 1 \mathrm{H}), 2.34(\mathrm{~s}, 3 \mathrm{H})$ ESI-MS: m/z Calculated 397.88, found [M+H] 398.8; Anal. Calcd for $\mathrm{C}_{19} \mathrm{H}_{16} \mathrm{ClN}_{5} \mathrm{OS}$ : C, 57.35; H, 4.05; Cl, 8.91; N, 17.60; O, 4.02; S, 8.06; found $\mathrm{C}, 57.65 ; \mathrm{H}, 4.26 ; \mathrm{N}, 17.78 ; \mathrm{S}, 8.21$. 
2-(2-(6-chloroquinolin-4-yl)hydrazinyl)-N-(6-fluorobenzo[d]thiazol-2-yl)acetamide (5c): mp 165-167 ${ }^{\circ} \mathrm{C}$; IR (KBr) $\left(v_{\max }, \mathrm{cm}^{-1}\right): 3080(\mathrm{C}-\mathrm{H}$ Aromatic), $3059(\mathrm{C}-\mathrm{H}$ alkene $), 1655(\mathrm{C}=\mathrm{C}$ alkene), $1550\left(\mathrm{C}=\mathrm{C}\right.$ aromatic), $1680\left(\mathrm{C}=\mathrm{O}\right.$ amide), 1270 (C-N Aromatic) ${ }^{1} \mathrm{H}$ NMR (400 MHz, DMSO) $\delta 11.07(\mathrm{~s}, 1 \mathrm{H}), 8.56(\mathrm{~d}, 1 \mathrm{H}), 8.04(\mathrm{dd}, 1 \mathrm{H}), 7.99-7.91(\mathrm{~m}, 2 \mathrm{H}), 7.59(\mathrm{~d}, 1 \mathrm{H}), 7.53(\mathrm{dd}$, $1 \mathrm{H}), 7.26(\mathrm{td}, 1 \mathrm{H}), 6.68(\mathrm{~d}, 1 \mathrm{H}), 3.57(\mathrm{~s}, 1 \mathrm{H}), 3.50(\mathrm{~s}, 2 \mathrm{H}), 3.09(\mathrm{~s}, 1 \mathrm{H})$; ESI-MS: m/z Calculated 401.85, found $[\mathrm{M}+\mathrm{H}]^{+}$402.8; Anal. Calcd for $\mathrm{C}_{18} \mathrm{H}_{13} \mathrm{ClFN}_{5} \mathrm{OS}: \mathrm{C}, 53.80 ; \mathrm{H}, 3.26 ; \mathrm{Cl}, 8.82 ; \mathrm{F}, 4.73$; N, 17.43; O, 3.98; S, 7.98; found C, 53.97; H, 3.45; N, 17.61; S, 7.87.

2-(2-(6-chloroquinolin-4-yl)hydrazinyl)-N-(6-methoxybenzo[d]thiazol-2-yl)acetamide (5d): IR $(\mathrm{KBr})\left(v_{\max }, \mathrm{cm}^{-1}\right): 3045(\mathrm{C}-\mathrm{H}$ Aromatic), $3076(\mathrm{C}-\mathrm{H}$ alkene $), 1646(\mathrm{C}=\mathrm{C}$ alkene), $1530(\mathrm{C}=\mathrm{C}$ aromatic), 1677 (C=O amide), 1289 (C-N Aromatic); ${ }^{1} \mathrm{H}$ NMR (400 MHz, DMSO) $\delta 10.32$ (s, $\left.1 \mathrm{H}\right)$, $8.66(\mathrm{~d}, 1 \mathrm{H}), 8.04-7.92(\mathrm{~m}, 3 \mathrm{H}), 7.89(\mathrm{~d}, 1 \mathrm{H}), 7.60(\mathrm{dd}, 1 \mathrm{H}), 7.24(\mathrm{dd}, 1 \mathrm{H}), 6.67(\mathrm{~d}, 1 \mathrm{H}), 3.79(\mathrm{~d}$, 4H), $3.61(\mathrm{~s}, 1 \mathrm{H}), 3.36(\mathrm{~s}, 1 \mathrm{H}), 0.70(\mathrm{~s}, 1 \mathrm{H})$; ESI-MS: m/z Calculated 413.88, found $[\mathrm{M}+\mathrm{H}]^{+} 414.8$; Anal. Calcd for $\mathrm{C}_{19} \mathrm{H}_{16} \mathrm{ClN}_{5} \mathrm{O}_{2} \mathrm{~S}$ : C, 55.14; H, 3.90; Cl, 8.57; N, 16.92; O, 7.73; S, 7.75; found C, $55.46 ; \mathrm{H}, 4.20 ; \mathrm{N}, 16.78 ; \mathrm{S}, 7.89$.

2-(2-(6-chloroquinolin-4-yl)hydrazinyl)-N-(6-nitrobenzo[d]thiazol-2-yl)acetamide (5e):

IR $(\mathrm{KBr})\left(v_{\max }, \mathrm{cm}^{-1}\right): 3056(\mathrm{C}-\mathrm{H}$ Aromatic), $3046(\mathrm{C}-\mathrm{H}$ alkene $), 1676(\mathrm{C}=\mathrm{C}$ alkene $), 1590(\mathrm{C}=\mathrm{C}$ aromatic), $1665\left(\mathrm{C}=\mathrm{O}\right.$ amide), 1310 (C-N Aromatic); ${ }^{1} \mathrm{H}$ NMR (400 MHz, DMSO) $\delta 8.91(\mathrm{t}, 1 \mathrm{H})$, $8.81(\mathrm{~s}, 1 \mathrm{H}), 8.64(\mathrm{~d}, 1 \mathrm{H}), 8.26-8.15(\mathrm{~m}, 2 \mathrm{H}), 8.02(\mathrm{~d}, 1 \mathrm{H}), 7.61-7.52(\mathrm{~m}, 2 \mathrm{H}), 6.69(\mathrm{~d}, 1 \mathrm{H})$, $3.44(\mathrm{~s}, 1 \mathrm{H}), 3.35(\mathrm{~s}, 1 \mathrm{H}), 3.28(\mathrm{~s}, 1 \mathrm{H}), 1.38(\mathrm{~s}, 1 \mathrm{H})$; ESI-MS: $\mathrm{m} / \mathrm{z}$ Calculated 428.85, found [M+H] ${ }^{+}$429.8; Anal. Calcd for $\mathrm{C}_{18} \mathrm{H}_{13} \mathrm{ClN}_{6} \mathrm{O}_{3} \mathrm{~S}$ : C, 50.41; H, 3.06; Cl, 8.27; N, 19.60; O, 11.19; S, 7.48; found $\mathrm{C}, 50.76 ; \mathrm{H}, 3.35 ; \mathrm{N}, 19.89 ; \mathrm{S}, 7.65$.

N-(6-chlorobenzo[d]thiazol-2-yl)-2-(2-(6-chloroquinolin-4-yl)hydrazinyl)acetamide (5f):

IR $(\mathrm{KBr})\left(\mathrm{v}_{\max }, \mathrm{cm}^{-1}\right): 3076(\mathrm{C}-\mathrm{H}$ Aromatic), $3092(\mathrm{C}-\mathrm{H}$ alkene $), 1678(\mathrm{C}=\mathrm{C}$ alkene $), 1556(\mathrm{C}=\mathrm{C}$ aromatic), $1679\left(\mathrm{C}=\mathrm{O}\right.$ amide), 1340 (C-N Aromatic); ${ }^{1} \mathrm{H}$ NMR (400 MHz, DMSO) $\delta 11.04(\mathrm{~s}, 1 \mathrm{H})$, $8.55(\mathrm{~d}, 1 \mathrm{H}), 8.24(\mathrm{~d}, 1 \mathrm{H}), 7.96(\mathrm{dd}, 2 \mathrm{H}), 7.61-7.48(\mathrm{~m}, 3 \mathrm{H}), 6.68(\mathrm{~d}, 1 \mathrm{H}), 3.56(\mathrm{~s}, 1 \mathrm{H}), 3.49(\mathrm{~s}$, 2H), 3.08 (s, 1H); ESI-MS: $\mathrm{m} / \mathrm{z}$ Calculated 418.30, found $[\mathrm{M}+\mathrm{H}]{ }^{+} 419.3$; Anal. Calcd for $\mathrm{C}_{18} \mathrm{H}_{13} \mathrm{Cl}_{2} \mathrm{~N}_{5} \mathrm{OS}$ : C, 51.68; H, 3.13; Cl, 16.95; N, 16.74; O, 3.82; S, 7.67; found $\mathrm{C}, 51.86 ; \mathrm{H}, 3.42$; N, 16.98; S, 7.87.

\section{2-(2-(6-chloroquinolin-4-yl)hydrazinyl)-N-(6-ethoxybenzo[d]thiazol-2-yl)acetamide (5g):}

IR $(\mathrm{KBr})\left(\mathrm{v}_{\max }, \mathrm{cm}^{-1}\right): 3090(\mathrm{C}-\mathrm{H}$ Aromatic), $3092(\mathrm{C}-\mathrm{H}$ alkene), $1672(\mathrm{C}=\mathrm{C}$ alkene $), 1572(\mathrm{C}=\mathrm{C}$ aromatic), 1650 (C=O amide), 1348 (C-N Aromatic); ${ }^{1} \mathrm{H}$ NMR (400 MHz, DMSO) $\delta 10.41(\mathrm{~s}, 1 \mathrm{H})$, $8.71(\mathrm{~d}, 1 \mathrm{H}), 8.03-7.95(\mathrm{~m}, 3 \mathrm{H}), 7.73(\mathrm{~d}, 1 \mathrm{H}), 7.63(\mathrm{dd}, 1 \mathrm{H}), 7.23(\mathrm{dd}, 1 \mathrm{H}), 6.70(\mathrm{~d}, 1 \mathrm{H}), 4.03(\mathrm{q}$, 2H), $3.66(\mathrm{~s}, 1 \mathrm{H}), 3.59(\mathrm{~s}, 1 \mathrm{H}), 2.99(\mathrm{~s}, 1 \mathrm{H}), 1.43$ - $1.35(\mathrm{~m}, 4 \mathrm{H})$; ESI-MS: m/z Calculated 427.91, found $[\mathrm{M}+\mathrm{H}]^{+}$428.91; Anal. Calcd for $\mathrm{C}_{20} \mathrm{H}_{18} \mathrm{ClN}_{5} \mathrm{O}_{2} \mathrm{~S}$ : C, 56.14; H, 4.24; Cl, 8.29; N, 16.37; O, 7.48; S, 7.49; found C, 56.48; H, 4.46; N, 16.64; S, 7.78.

\section{Determination of Antibacterial Activity}

Antibacterial activities of 5a-5g were carried out in Nutrient-agar plates by well diffusion assay. Cultures were activated in Nutrient broth. Isolates were inoculated in Nutrient broth and incubated at $37^{\circ} \mathrm{C}$ for 24 hours for activation of cultures and then centrifuged at $3000 \mathrm{rpm}$ for $15 \mathrm{~min}$ and the supernatant was collected to study antibacterial activity.

Using in-vitro agar well diffusion method, antimicrobial activity experiments were carried out. The activity of 5a-5g against test microorganisms (1000 $\mu 1$ volume with $1000 \mu \mathrm{g} / \mathrm{ml}$ concentration) of activated test cultures 2 Gram negative and 2 Gram positive; viz. Enterobacter aerogens MTCC No. 8558, Escherichia coli MTCC No. 1610, Micrococcus luteus MTCC No. 11948 and Bacillus cereus MTCC No. 8557) was inoculated in molten agar and poured into sterile plates than allowed to solidify. Wells with $5 \mathrm{~mm}$ diameter were prepared at equal distance in solidified agar plates using cup-borer. Various derivatives $4 \mathrm{a}-41$ with $1000 \mu \mathrm{gm} / \mathrm{ml}$ concentration were inoculated in the wells 
of nutrient agar whereas test microorganisms were inoculated by pour plate technique. The plates were incubated at $37^{\circ} \mathrm{C}$ for 24 hours. The inhibition zones were measured at the end of the incubation period.

\section{RESULT AND DISCUSSION}

\section{Chemistry}

The synthetic pathway for preparation of different derivative of N-(benzo[d]thiazol-2-yl)-2(2-(6-chloroquinolin-4-yl)hydrazinyl)acetamide is shown in Scheme 1. To explore the scope and limitations of this reaction further, we extended our studies to the use of various substituted 2aminobenzothiazole and chloroacetylchloride in the presence of diethyl amine. It was gratifying to observe that most of the tested substrates exhibited satisfactory reactivity profiles, in all cases leading to a linkage sequence such as hydrazine hydrate and 4,7-dichloroquinoline that readily afforded the target structures (Table 1).

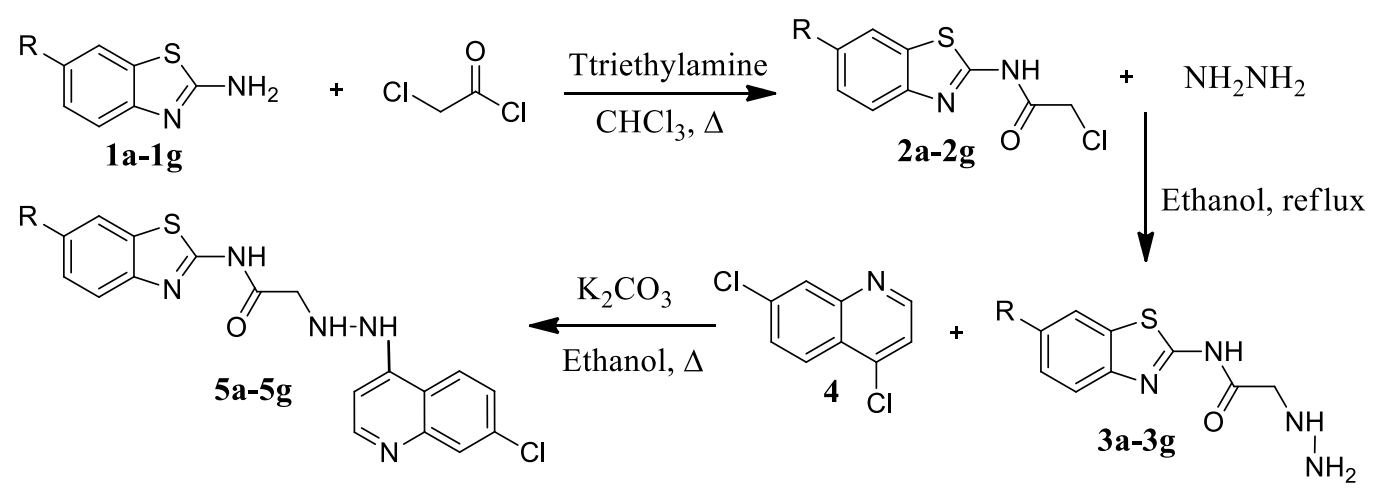

Scheme 1 synthsis of Targeted compound 
Table 1 Synthesis of N-(benzo[d]thiazol-2-yl)-2-(2-(6-chloroquinolin-4-yl)hydrazinyl) acetamide derivative $5 \mathrm{a}-5 \mathrm{~g}$

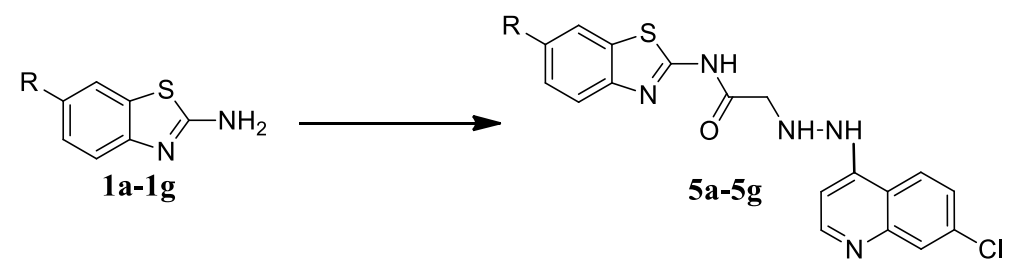

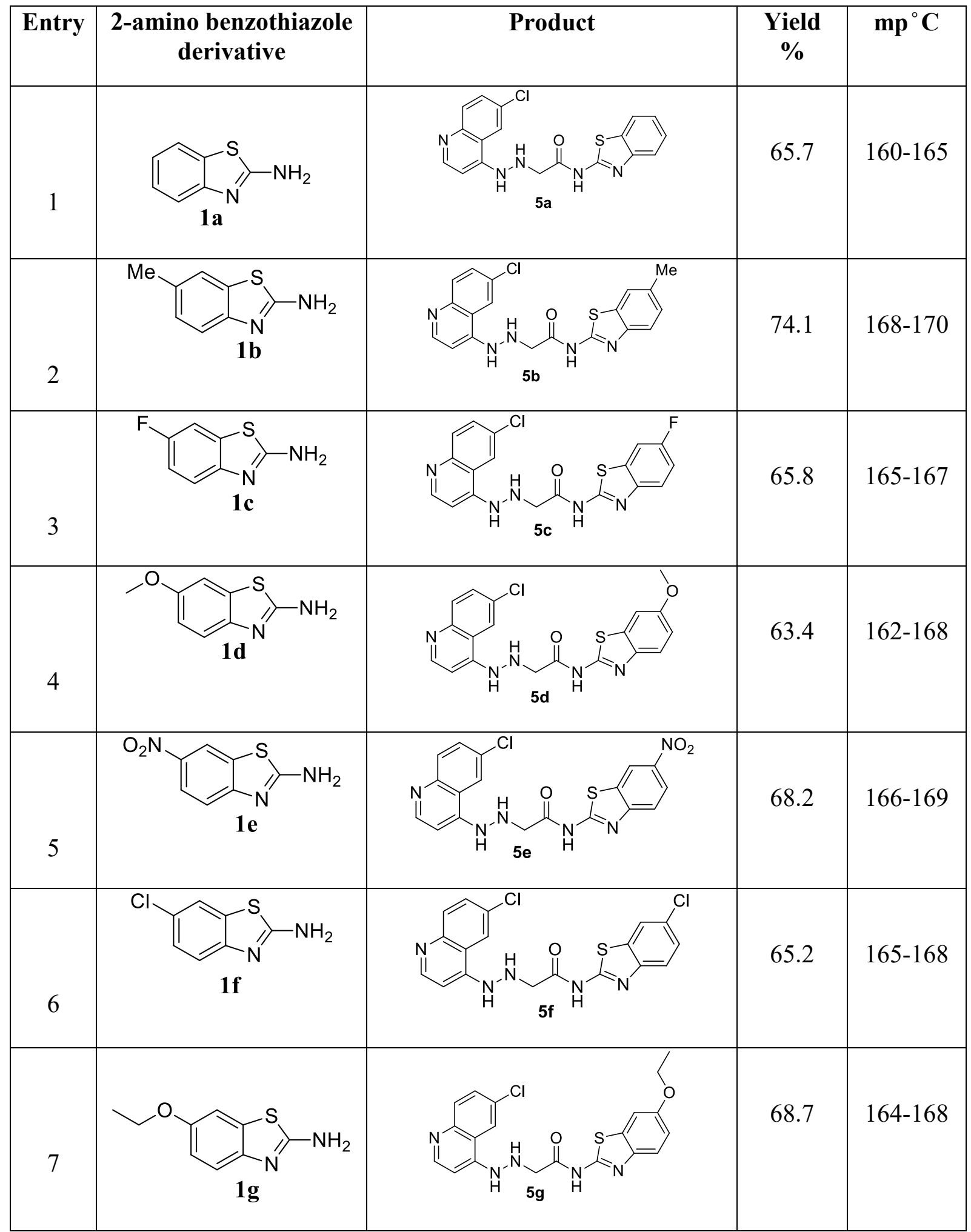


A series of compound $\mathbf{5 a}-\mathbf{5 g}$ has been synthesized by conventional method as illustrated in scheme 1. The structures of all the newly synthesized compounds were confirmed by elemental analysis and FT-IR, ${ }^{1} \mathrm{H}$ NMR, and Mass analysis. The IR spectra of compounds 5a shows six main peaks at 3070 (C-H Aromatic), 3034 ( $\mathrm{C}-\mathrm{H}$ alkene), $1640(\mathrm{C}=\mathrm{C}$ alkene), $1540(\mathrm{C}=\mathrm{C}$ aromatic), 1670 ( $\mathrm{C}=\mathrm{O}$ amide), 1390 (C-N Aromatic) respectively. In ${ }^{1} \mathrm{H}$ NMR spectrum of 5a shows characteristic peaks at $8.69 \delta$ value resulting from - $\mathrm{NH}$ of amide group. Aromatic proton of targeted compound resonates at 8.47 $-6.73 \delta \mathrm{ppm}$ and Two $-\mathrm{NH}$ proton resonate at 3.22, $1.83 \delta$. The mass spectrum of selected compound 5a showed $[\mathrm{M}+\mathrm{H}]^{+}$peak at $\mathrm{m} / \mathrm{z}$ 384.8. The appearance of a molecular ion peak at 384.8 mass unit supports the structure of compound $\mathbf{5 a}$.

\section{Antibacterial Activity}

The antibacterial activity of the synthesized compound was carried out on Nutrient-agar plates by well-diffusion assay against test culture. Cultures were activated in Nutrient broth. Isolates inhibits the above mentioned organisms or not were studied and zone of inhibition was measured in terms of zone diameter and with the help of that zone index was calculated where streptomycin was used as standard.

\section{Determination of activity index}

The activity index of the probiotic culture was calculated as:

$$
\text { Activity index }(\text { A.I. })=\frac{\text { Mean of zone of inhibition of derivative }}{\text { Zone of inhibition obtained for standard antibiotic drug }}
$$

Note: Standard drug used was Streptomycin with $1000 \mu \mathrm{g} / \mathrm{ml}$ concentration

The synthesized compounds $\mathbf{5 a - 5 g}$ were tested in vitro for antibacterial activity against Gramnegative Enterobacter aerogens (MTCC No. 8558), Escherichia coli (MTCC No. 1610), and Grampositive Micrococcus luteus (MTCC No. 11948), Bacillus cereus (MTCC No. 8558) by measuring the zone of inhibition in $\mathrm{mm}$. Antibacterial screening results (the zone of inhibition), presented in Table 2, revealed that all compounds tested showed some degree of antibacterial activity. Compound $\mathbf{5 a}, \mathbf{5 b}$ and $\mathbf{5 g}$ have showed very close activity to standard drug against Bacillus cereus gram positive bacteria. When compound $\mathbf{5 b}, \mathbf{5 c}, \mathbf{5 d}, \mathbf{5 f}$, and $5 \mathrm{~g}$ also shows parallel, or more activity to standard drug against Micrococcus luteus gram positive bacteria. The antibacterial activity of compounds 5c-5g were more than 83\% inhibition than the standard against Enterobacter aerogens bacteria. Compounds $\mathbf{5 b}, \mathbf{5 e}, \mathbf{5 f}$, and $\mathbf{5 g}$ more pronounced activity compared to streptomycin against Escherichia coli bacteria.

Table 2 Antibacterial Activity of synthesized compounds 5a-5g

\begin{tabular}{|c|c|c|c|c|c|c|c|c|}
\hline \multirow[b]{2}{*}{ 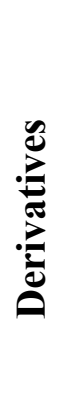 } & \multicolumn{2}{|c|}{$\begin{array}{c}\text { Enterobacter aerogens } \\
\text { MTCC No. } 8558\end{array}$} & \multicolumn{2}{|c|}{$\begin{array}{l}\text { Escherichia coli } \\
\text { MTCC No. } 1610\end{array}$} & \multicolumn{2}{|c|}{$\begin{array}{l}\text { Micrococcus luteus } \\
\text { MTCC No. } 11948\end{array}$} & \multicolumn{2}{|c|}{$\begin{array}{c}\text { Bacillus cereus } \\
\text { MTCC No. } 8558\end{array}$} \\
\hline & $\begin{array}{l}\text { Mean value } \\
\text { for Zone of } \\
\text { Inhibition } \\
\quad(\mathrm{mm})\end{array}$ & $\begin{array}{l}\text { Activity } \\
\text { Index } \\
\text { (A.I.) }\end{array}$ & $\begin{array}{c}\text { Mean } \\
\text { value for } \\
\text { Zone of } \\
\text { Inhibition } \\
(\mathrm{mm})\end{array}$ & $\begin{array}{c}\text { Activity } \\
\text { Index } \\
\text { (A.I.) }\end{array}$ & $\begin{array}{c}\text { Mean } \\
\text { value for } \\
\text { Zone of } \\
\text { Inhibition } \\
\text { (mm) }\end{array}$ & $\begin{array}{c}\text { Activity } \\
\text { Index } \\
\text { (A.I.) }\end{array}$ & $\begin{array}{c}\text { Mean } \\
\text { value for } \\
\text { Zone of } \\
\text { Inhibition } \\
(\mathrm{mm})\end{array}$ & $\begin{array}{c}\text { Activity } \\
\text { Index } \\
\text { (A.I.) }\end{array}$ \\
\hline $5 a$ & 21 & 0.875 & 19 & 0.791 & 19 & 0.791 & 18 & 0.750 \\
\hline $5 b$ & 22 & 0.916 & 17 & 0.708 & 20 & 0.833 & 24 & 1.00 \\
\hline $5 c$ & 19 & 0.791 & 20 & 0.833 & 24 & 1.00 & 23 & 0.958 \\
\hline
\end{tabular}




\begin{tabular}{|c|c|c|c|c|c|c|c|c|}
\hline $\mathbf{5 d}$ & 20 & 0.833 & 21 & 0.875 & 26 & 1.08 & 16 & 0.666 \\
\hline $\mathbf{5 e}$ & 23 & 0.958 & 26 & 1.08 & 18 & 0.75 & 19 & 0.791 \\
\hline $\mathbf{5 f}$ & 24 & 1.000 & 24 & 1.08 & 21 & 0.875 & 18 & 0.750 \\
\hline $\mathbf{5 g}$ & 25 & 1.041 & 22 & 0.916 & 24 & 1.00 & 21 & 0.875 \\
\hline $\begin{array}{c}\text { Std } \\
\mathbf{d r u g}\end{array}$ & 24 & --- & 24 & --- & 24 & --- & 24 & --- \\
\hline
\end{tabular}

\section{CONCLUSION}

Summarizing, a series of N-(benzo[d]thiazol-2-yl)-2-(2-(6-chloroquinolin-4-yl)hydrazinyl) acetamide derivative have been synthesized by sequencing reaction of 2-aminobenzothiazole with chloroacetylchloride, hydrazine hydrate and 4,7-dichloroquinoline. It has been synthesized successfully in appreciable yields and screened for their in vitro antibacterial activity. From the antibacterial activity study, Compounds $\mathbf{5 b}, \mathbf{5 c}, \mathbf{5 d}$ and $\mathbf{5 g}$ emerged as the most active antibacterial activity against gram positive bacteria. Moreover, Compounds $\mathbf{5 c}$, $\mathbf{5 f}$ and $\mathbf{5 g}$ arose as the most active antibacterial activity against gram negative bacteria. As a consequence, we can conclude that newly synthesized derivatives can be used for the development of new antibacterial drugs to cure many disorders caused by the different bacterial species.

\section{Acknowledgement}

The authors are thankful to UGC-Info net and INFLIBNET Gujarat University for providing e-source facilities. Both Mayuri A. Borad and Manoj N. Bhoi is thankful to UGC-BSR fellowship for financial assistance.

\section{References}

[1] AT. Balaban, DC. Oniciu, AR. Katritzky, Aromaticity as a cornerstone of heterocyclic chemistry, Chemical reviews 104 (2004) 2777-2812.

[2] T. Torroba, Poly-sulfur-nitrogen heterocycles via sulfur chlorides and nitrogen reagents, Journal für praktische Chemie 341 (1999) 99-113.

[3] N. Nayeem, G. Denny, Synthesis of some Mannich bases from sulphonamides and benzothiazole derivatives and evaluation of their anti tubercular activity and anti microbial activity, Der Pharma Chemica 4 (2012) 1277-82.

[4] A. Geronikaki, P. Vicini, N. Dabarakis, A. Lagunin, V, Poroikov, J, Dearden, et al. Evaluation of the local anaesthetic activity of 3-aminobenzo [d] isothiazole derivatives using the rat sciatic nerve model, Eur. J. Med. Chem. 44 (2009) 473-481.

[5] G. Mariappan, P. Prabhat, L. Sutharson, J. Banerjee, U. Patangia, S. Nath, Synthesis and antidiabetic evaluation of benzothiazole derivatives, Journal of the Korean Chemical Society 56 (2012) 251-256.

[6] D. Pareek, M. Chaudhary, PK. Pareek, R. Kant, KG. Ojha, R. Pareek, et al. Synthesis of some bioactive 4-thiazolidinone derivatives incorporating benzothiazole moiety, Der Chemica Sinica 1 (2010) 22-35.

[7] P. Arora, S. Das, M. Ranawat, N. Arora, M. Gupta, Synthesis and biological evaluation of some novel chromene-2-one derivatives for antipsychotic activity, J Chem Pharm Res 2 (2010) 317-323. 
[8] RK. Gill, PMS. Bedi, Synthesis and cytotoxic evaluation of 2- aminobenzothiazole derivatives, International Journal of Natural Product Science 1 (2012) 100.

[9] MI. Nessim, et al, The effect of some benzothiazole derivatives as antioxidants for base stock, International Journal of Current Research, 5 (2013) 1111-1117.

[10] H. Kaur, S. Kumar, I, Singh, K. Saxena, A. Kumar, Synthesis, characterization and biological activity of various substituted benzothiazole derivatives, Dig J Nanomater Bios 5 (2010) 67-76.

[11] MA. Mahran, S. William, F. Ramzy, AM. Sembel, Synthesis and in vitro evaluation of new benzothiazole derivatives as schistosomicidal agents, Molecules 12 (2007) 622-633.

[12] F. Delmas, C. Di Giorgio, M. Robin, N. Azas, M. Gasquet, C. Detang, et al. In vitro activities of position 2-substitution-bearing-6-nitro-and 6-amino-benzothiazoles and their corresponding anthranilic acid derivatives against Leishmania infantum and Trichomonas vaginalis, Antimicrobial agents and chemotherapy 46 (2002) 2588-2594.

[13] R. Singh, S. Sharma, Synthesis, characterization and evaluation of 2-imino benzothiazole derivatives as anticonvulsant agents, Int J Pharma Sci Res 5 (2014) 213-217.

[14] M. Mahran, S. El-Nassry, S. Allam, L. El-Zawawy, Synthesis of some new benzothiazole derivatives as potential antimicrobial and antiparasitic agents, Die Pharmazie-An International Journal of Pharmaceutical Sciences 58 (2003) 527-530.

[15] MS. Yar, ZH. Ansari, Synthesis and in vivo diuretic activity of biphenyl benzothiazole-2carboxamide derivatives, Acta Pol Pharm-Drug Res 66 (2009) 387-392.

[16] D Mahajan, J. Bhosale, R. Bendre, Synthesis, characterization and plant growth regulator activity of some substituted 2-amino benzothiazole derivatives, J Appl Chem 2 (2013) 765-771.

[17] D. Munirajasekhar, M. Himaja, V. Mali Sunil, Synthesis and Anthelmintic activity of 2Amino-6-substituted Benzothiazoles, Int Res J Pharm 2 (2011) 114-117.

[18] M. Chaudhary, D. Pareek, PK. Pareek, R. Kant, K. Ojha, A. Pareek, Synthesis of some biologically active benzothiazole derivatives, Der Pharma Chemica 2 (2010) 281-293.

[19] A. Marella, OP. Tanwar, R. Saha, MR. Ali, S. Srivastava, M. Akhter, et al. Quinoline: A versatile heterocyclic, Saudi Pharmaceutical Journal 21 (2013) 1-12.

[20] M. Amir, S. Asif, I. Ali, MZ. Hassan, Synthesis of benzothiazole derivatives having acetamido and carbothioamido pharmacophore as anticonvulsant agents, Med. Chem. Res. 21 (2012) 26612670 . 